\title{
Tympanic Membrane Derived Stem Cell-Like Cultures for Tissue Regeneration
}

\author{
Lawrence J. Liew,1,2 Linda Q. Chen,2,3 Allen Y. Wang,1,2,4 Magnus von Unge,5,6 \\ Marcus D. Atlas, 1,2 and Rodney J. Dilley1,2,7
}

Epidermal cells with stem cell-like characteristics have been identified in the tympanic membrane (TM) and localized specifically to the umbo and annulus regions. While they have been proposed to play a role in the regeneration of both acute and chronic TM perforations, evidence for the mechanism and regulation of their contribution is not yet described. Indeed, the behavior of these putative stem cells is largely unknown, in part due to a lack of refined methods for efficient cell isolation. In this study, we compared different explant techniques using normal and perforated rat TM tissues and investigated their ex vivo characteristics. TM after perforation in vivo showed increased staining for epidermal stem cell markers integrin b1 and cytokeratin (CK) 19, and for proliferation Ki-67, indicating activation of the proliferative centers. A mixed population of fibroblasts and epithelial cells were isolated from explant cultures. Excised TM umbo implanted on a culture well insert was the most effective technique. Explants made from perforated TM produced cells before those from unperforated TM. More importantly, the implanted TM umbo organoid was capable of producing cells in a continuous manner, allowing subsequent harvest using trypsin. Primary rat TM epithelial cell cultures positive for pancytokeratin had colony forming activity and could be enriched for CK 19-positive cells that were capable of culture expansion by proliferation and cell migration when subject to a wound assay. Taken together, trauma to the TM activated the proliferative centers and prompted early cell production from TM umbo organoid cultures, which produced TM stem cell-like cultures that proved suitable for tissue engineering of the TM.

Keywords: tympanic membrane, epidermal stem cells, explant culture, integrin b1, cytokeratin 19, culture well

inserts

Introduction

$\mathrm{T}$ he tympanic membrane (TM) is a thin, fibrocellular

sheet that separates the outer ear from the middle ear. It consists of two main parts: the thinnest and stiffest part, the pars tensa, which responds to sound pressure, and the pars flaccida, which is more distensible. The TM is covered by a keratinized stratified squamous epithelium on the lateral (ear canal) side and a mucosal epithelium on the medial (middle ear) side. Between these epithelia is a substantial middle fibrous layer with specialized radial and circular collagen fiber architecture [1,2]. A thickened fibrous ring-like structure, the annulus, lies at the periphery of the TM, suspending it from the bony ear canal. At the center of the TM is a strong attachment to the handle of malleus and at the tip of this structure, the umbo, is a proliferative center recognized for substantial keratinocyte production [3-5].

Tympanic membrane perforation (TMP) is a prevalent condition worldwide associated commonly with otitis media. While most TMPs heal spontaneously without treatment, some may progress to chronic wounds (which fail to heal for 3 months or longer). Chronic TMPs are associated with aural suppuration and conductive hearing loss. Meanwhile, the healing of acute TMPs is a complex yet rapid process where hyperplastic epithelial cells (keratinocytes) initially migrate across the TMP to close the outer layer, followed by fibrous tissue formation to restore the middle layer and lastly, the healing process is completed with the proliferation of mucosal cells [6-8]. More recently, the role 
of stem cells has been implicated in the maintenance and healing of TM $[3,9,10]$.

Various "ink-dot"' studies [11-13] have provided insight into a unique migration pattern of the epidermal layer of the $\mathrm{TM}$, in which a superficial ink stain was observed to migrate

1Ear Sciences Centre, School of Medicine, University of Western Australia, Perth, Australia.

${ }_{2}$ Ear Science Institute Australia, Perth, Australia.

3 School of Veterinary and Life Sciences, Murdoch University, Perth, Australia.

4Department of Otolaryngology, Head and Neck, Skull Base Surgery, Sir Charles Gairdner Hospital, Perth, Australia.

5Akershus University Hospital and University of Oslo, Oslo, Norway.

${ }_{6}$ Centre for Clinical Research Va“stera ${ }^{\circ}$, University of Uppsala, Uppsala, Sweden.

7The Centre for Cell Therapy and Regenerative Medicine, School of Medicine, University of Western Australia, Perth, Australia.

in a radial/helical pattern toward the TM periphery and ear canal. This migratory pattern indicates that in normal TM homeostasis the "regenerative region" at the umbo produces new keratinocytes that subsequently migrate peripherally to maintain the cell population.

To date, little is known on the biology of cells in the TM umbo. Knutsson et al. have suggested that interfollicular progenitor or stem cells may be the predominant cell type that populates the TM, which lacks hair follicles and glands [3]. Two studies have also reported potential stemcell populations localized to the umbo, handle of malleus and annular regions of rat TM [5,10], with evidence including immunohistochemical staining for cell surface antigens such as integrins a6 and b1 or cytokeratin (CK) 19. Moreover, it appeared that these putative TM stem cells were involved in the regeneration of perforated TM regardless of acute or chronic status [10]. Currently, there are no confirmed cell surface markers for TM-derived epidermal stem cells. Unlike the well-defined mesenchymal stromal cells [14], there appears to be a lack of minimal defining characteristics for epidermal stemcells [15]. Cultivation of human TM-derived keratinocytes has been reported [16] but there are no studies specifically reporting on isolation or cultivation of TM-derived stem cells in vitro. It remains unclear whether terminally differentiated keratinocytes may be a suitable in vitro model to evaluate the biology and healing mechanism of TMPs or whether stem cells like those in the umbo are more valuable. In this study, we explored different methodologies to isolate and enrich potential TM stem cells. TMs were harvested for histological evaluation and explant culture. Isolated cells in culture were enriched and characterized for putative epidermal stem cell integrin b1, CK 19, and proliferative marker Ki-67.

\section{Materials and Methods}

Animals

Male Sprague-Dawley rats $(\mathrm{n}=40)$, weighing 200-250 g, were obtained from Animal Resources Centre (Murdoch, Western Australia, Australia). This study was approved by the University of Western Australia Animal Ethics Committee (No. 100/1249). All experiments were performed in accordance with the Australian Code of Practice for the Care and Use of Animals for Scientific Purposes. The rats were maintained in a room with 12-h light/dark cycles and with food and water available ad libitum. Animals were examined and imaged using a video-otomicroscope (MedRX) to exclude middle ear infection before the experiment. All animals 
were euthanized by intraperitoneal administration of pentobarbitone $(160 \mathrm{mg} / \mathrm{kg})$. Both left and right external ears were

removed at the osteocartilaginous junctions, and the tympanic bulla was isolated. The bulla bone was then shaved with a scalpel blade to expose the TM to surgical harvest of tissues.

\section{Myringotomy}

To study the effects of physical trauma on the proliferative centers of the TM (umbo and annulus), rats underwent myringotomy and were sacrificed 3 days later as acute TMP. Briefly, rats were anesthetized with isoflurane (Bomac, New Zealand) (4\% induction, $2 \%$ maintenance in $100 \%$ oxygen) and myringotomy was performed bilaterally via a transcanal approach using a Wullstein needle. The posterior half of the pars tensa was perforated to a diameter of $0.8 \mathrm{~mm}$, gauged using the needle tip diameter.

\section{Processing of rat TM}

The TMs were either used to generate explant cultures or fixed in 10\% neutral buffered formalin for histological analysis. For tissue culture, freshly extracted TMs were rinsed thoroughly in $2 \mathrm{~mL}$ of phosphate-buffered saline (PBS) containing $1 \%$ penicillin-streptomycin (Gibco, Grand Island, NY) and transferred to a $35 \mathrm{mmdiameter}$ culture dish containing $1 \mathrm{mLof}$ $0.25 \%$ Dispase II solution (Gibco). Following overnight incubation at 4_C, the TMs were peeled off fromthe bony bulla and separated from the external ear canal skin using a pair of forceps. Peeled rat TM were used to generate explant cultures.

\section{Histology and immunohistochemistry of rat TM}

After $24 \mathrm{~h}$ fixation, TM were decalcified in $14 \%$ ethylenediaminetetraacetic acid (EDTA; Sigma Aldrich, St. Louis,

MO) solution ( $\mathrm{pH} 7.2$ ) at 37_C for 10 days, then processed routinely for paraffin histology and immunohistochemistry. Processed TM were sectioned at $5 \mathrm{~mm}$ and stained with hematoxylin and eosin (H\&E) and digitally scanned using an Aperio ScanScope XT (20 • /0.75 Plan Apo objective; Leica Biosystems, Nußloch, Heidelberg). For immunohistochemistry, TM sections were treated in $3 \% \mathrm{H}_{2} \mathrm{O}_{2}$ for $10 \mathrm{~min}$ to block endogenous peroxidase activity, followed by Rodent $\mathrm{R}$ blocker (Biocare, Concord, CA) for 30min at room temperature (RT). The sections were then stained with rabbit anti Integrin b1 (Novus Biologicals, Littleton, CO), rabbit anti CK 19 (Novus Biologicals), mouse anti-Ki-67 antibody (Biocare), and mouse anti-pancytokeratin antibody (Biocare) at 1:100 dilution. Sections were rinsed twice in PBS and incubated with HRP polymer labeled secondary antibody (Mouse-onRodent; Biocare) for 30min at RT. DAB (Steady DAB/Plus; Abcam, Cambridge, United Kingdom) was used for visualization of staining. The nuclei were counterstained with hematoxylin and digitally scanned as above.

\section{Cell culture}

Rat TM explant cultures were generated using three different methods:

1. WholeTMwas adhered to the base of a $35 \mathrm{mmdiameter}$ tissue culture dish (Eppendorf, Hamburg, Germany) coated with $5 \mathrm{mg} / \mathrm{cm}_{2}$ collagen Type IV (human placenta; Sigma-Aldrich). Adherence method was by placing tissue in a scant drop of medium, then drying in 
a 37_C incubator for $15 \mathrm{~min}$. Once adhered, the tissue was re-wetted with $1 \mathrm{~mL}$ of complete serum-free keratinocyte medium (KSFM) containing human recombinant Epidermal Growth Factor 1-53, Bovine Pituitary Extract, and 1\% penicillin-streptomycin (Gibco) for ongoing culture.

2. Umbo region of the TM was excised and adhered onto coated $35 \mathrm{~mm}$ tissue culture dish as described above.

3. Excised TM umbo tissues were grown as organoid explant culture on tissue culture insert membrane as previously described [17].

Primary cell cultures were expanded further in subculture at 10 days or when primary outgrowth from explants became confluent. Briefly, cells were rinsed twice with $1 \mathrm{~mL}$ of PBS and incubated with $500 \mathrm{~mL}$ of Tryple Express (Gibco) for 15 min at 37_C. Tryple Express was neutralized by adding $2 \mathrm{~mL}$ of complete KSFM supplemented with $10 \%$ fetal bovine serum (FBS; Bovogen, East Keilor, Australia). Detached cells were centrifuged at $600 \mathrm{~g}$ for $5 \mathrm{~min}$ and replated in a collagen IV coated $25 \mathrm{~cm} 2$ cell culture flask (Greiner Bio-One, Frickenhausen, Germany). The cells were cultured in complete KSFM supplemented with $10 \%$ FBS for $48 \mathrm{~h}$ at 37 C before reducing FCS to $5 \%$ to suppress fibroblast proliferation. From the second passage onward, epidermal-like cells were cocultured at a 1:1 ratio with feeder cells, mitomycin-C (Sigma-Aldrich) treated mouse embryonic fibroblasts (MEF) at $2 \cdot 104$ cells per $\mathrm{cm} 2$.

Colony forming unit assay

Culture expanded TM epidermal-like cells were subjected to colony forming unit (CFU) assay. Briefly, confluent cells were detached from culture flasks using Tryple express (Gibco) and resuspended in $\mathrm{KSFM}+10 \% \mathrm{FBS}+1 \% \mathrm{P} / \mathrm{S}$. One thousand epidermal cells were seeded onto a $60 \mathrm{~mm}$ culture dish (Eppendorf) precultured with feeder cells ( $2 \cdot 104$ cells/ $\mathrm{cm}_{2}$ ). Cells were incubated for 10 days at 37_C/5\% $\mathrm{CO}_{2}$ with fresh media changed twice weekly. Cells were then fixed with $4 \%$ paraformaldehyde and stained with $0.05 \%$ Crystal violet for $20 \mathrm{~min}$. Dishes were evaluated under the microscope to determine the colony sizes.

Cell migration assay

Migration assays were performed using a two well silicon culture-insert system (Ibidi; Greiner Bio-One). Briefly, cells were seeded into the two cell reservoirs and incubated at 37 C $/ 5 \% \mathrm{CO}_{2}$ overnight for cells to attach and spread to confluence. A cell-free gap was then created by removing the insert and cell migration into the gap visualized by phase contrast microscopy. Images at 0,6 , and $18 \mathrm{~h}$ were captured using a digital camera.

Magnetic enrichment of potential epidermal stem cells Culture-expanded epidermal-like cells were trypsinized as previously described and a total of $1 \cdot 107$ cells were resuspended in wash buffer made of Hanks' Balanced Salt Solution (HBSS; Gibco)+0.2\% bovine serum albumin (BSA; Sigma-Aldrich). Rabbit anti CK 19 and integrin b1 (Novus Biologicals) were added to the suspension and incubated on ice for $2 \mathrm{~h}$. Cells were then rinsed and suspended in $80 \mathrm{~mL}$ of wash buffer with $20 \mathrm{~mL}$ of anti-rabbit IgG microbeads (Miltenyi Biotech, Teterow, Germany). Suspensions were further incubated for 40min on ice and magnetically selected using a 
Mini magnetic activated cell sorting (MACS) system and MS columns (Miltenyi Biotech). Cells were reseeded in a collagen IV coated glass bottom culture dish to confirm cell phenotype by immunocytochemistry or cryopreserved for later use.

Immunofluorescent staining for characterization of the primary cells

Cells were cultured on a collagen IV coated glass bottom 35mm dish (Greiner Bio-One) for 3-5 days at 37_C before immunofluorescent staining. The cells were rinsed with PBS and fixed with $4 \%$ paraformaldehyde for $10 \mathrm{~min}$ at RT. The cells were incubated with ice-cold $100 \%$ methanol for $10 \mathrm{~min}$ at -20_C before blocking with 5\% BSA for 30min at RT. Primary antibodies used were rabbit anti-CK 19 (1 in 100; Novus Biologicals), anti-vimentin (1 in 200; Abcam), and mouse anti-Ki-67 antibody ( 1 in 100; Biocare). Following overnight incubation at RT, the cells were rinsed three times with PBS $+0.01 \%$ Tween 20 before the addition of secondary antibodies: anti-mouse IgG Alexafluor 555 (1 in 300; Abcam), anti-rabbit IgG Alexafluor 555 (1 in 300; Abcam), and antimouse IgG Alexafluor 488 (Molecular Probes). The cells were incubated for $1 \mathrm{~h}$ at RT and then rinsed in PBS $+0.01 \%$ Tween 20 and counterstained with DAPI $(0.05 \mathrm{mg} / \mathrm{mL}$; Life Technologies $)$ for 20min. The cells were mounted in PBS:Glycerol (1:1) before viewing with an Olympus BX60 fluorescence microscope with appropriate fluorescence filters.

\section{Results}

Rat TM exhibits distinct trilaminar appearance

The rat TM had a morphology typical of mammals

(Fig. 1A), with pars tensa forming the majority of the structure, umbo at the center where the handle of the malleus is attached medially and a pars flaccida on the superior aspect. Cross sections of normal rat TM showed the prominent handle of malleus and well-organized trilaminar membrane throughout the pars tensa (Fig. 1B). The epidermal layer of TM showed a uniform but intense pancytokeratin staining and was thickened at the umbo (Fig. 1C). The middle fibrous and mucosal layers of the TM stained strongly for the fibroblast marker vimentin (Fig. 1D). Staining of putative epidermal stem cell markers Integrin b1 (Fig. 1E) and CK 19 (Fig. 1F) showed weak but uniform staining along the TM. Cellular proliferation in the normal rat TM was evaluated using Ki-67 staining and very few Ki-67-positive cells were found along the TM (Fig. 1G, black arrows heads) but more commonly seen in the glandular epithelium of ear canal skin (Fig. 1H, black arrows heads).

\section{Physical trauma activated the proliferative regions in rat $\mathrm{TM}$}

Rat TM postmyringotomy appeared opaque on the perforated side with visible blood vessels along the malleus and near the perforation site (Fig. 2A). A representative histological image showed thickening at both edges of the perforation, extending to annulus and umbo (Fig. 2B). At higher magnification, all three layers were seen to be thickened, with the epidermal layer most significantly thicker (margin outlined by black dashes) near perforation edges at the annulus

(Fig. 2C) and umbo (Fig. 2D). The expression of integrin b1

(Fig. 2E, F) and CK 19 (Fig. 2G, H) appeared elevated at the 
perforation edges. Occasional Ki-67-positive cells were seen in basal epidermal layers at the perforation site near the annular region (Fig. 2I) but a large number of Ki-67-positive cells were seen at the base (black arrow heads) of the epidermal layer near the handle of malleus (Fig. 2J).

Rat TM preserved its regenerative potential ex vivo Three explant culture techniques were explored here. In the whole rat TM explant culture (method 1), cell outgrowth from normal rat TM tissue was evident after 3 days in culture (Fig. 3A). In contrast, from perforated rat TM (myringotomy), outgrowth of a mixed cell population of fibroblasts and epidermal-like cells was seen as early as $24 \mathrm{~h}$ and a significantly larger mixed cell population was seen at the 3-day time point (Fig. 3B). Excised rat TM umbo tissues adhered to culture dish (method 2) showed cell outgrowth delayed to days 5-6 and cells then proliferated in a similar pattern as described. The majority of the cells displayed a cobblestone-like morphology (Fig. 3C) typical of epithelia. In method 3, the excised rat TM umbo tissue was placed in a small incision in a culture well insert membrane as an organoid explant culture (Fig. 3D). Cell outgrowth was seen as early as $24 \mathrm{~h}$ and confluent cells reaching the edges of the culture well insert within 10-14 days. Rat umbo tissues after outgrowth could be transplanted to new culture dishes (method 1-3) to generate further cultures but ceased proliferating after the third transfer. While tissue transfer was not performed in method 3 , multiple cell batches were harvested consecutively off the culture well insert membrane via in situ trypsinization. More importantly, cells were able to grow to confluence after trypsinization.

Rat TM epidermal-like cells could be expanded in culture and exhibit both clonogenicity and migrating ability

Primary cells isolated from all three types of explant cultures could be expanded using standard procedures. Upon re-plating, cells attached to collagen IV coated tissue culture plastic within a few hours and formed a heterogeneous cell population with a mixture of fibroblastlike and epidermal-like cells within 24-48 h. Fibroblast growth was suppressed by reducing serum content in the medium or a minimal trypsinization step, which selectively released fibroblasts from the culture surface. Enriched epidermal-like cells did not proliferate well and formed only very loose colonies upon fibroblast removal (Fig. 3E). From passage 2 onward, we introduced feeder cells (MEF) to the culture and epidermal-like cells were able to form large colonies (Fig. 3F). These epidermallike cells were slightly elongated. Both rat TM epidermal cells and human keratinocytes (HaCat) stained for pancytokeratin and Ki-67 (Fig. 5A, B). While the HaCats formed very distinct epithelial colonies (Supplementary

Fig. S1A; Supplementary Data are available online at www.liebertpub.com/scd), the rat cells formed looser colonies with bright-looking cytoplasm (Supplementary Fig. S1B) and had a higher nuclear to cytoplasm ratio under higher magnification (Supplementary Fig. S1C). Epidermal cells from rat TM were able to form colonies of 
$>100$ cells (Fig. 4A, B) in a CFU assay, indicating the presence of a stem cell-like population that was capable of undergoing extensive proliferation. In a scratch wound migration assay, rat TM epidermal cells were capable of closing the wound gap within $18 \mathrm{~h}$ (Fig. 4C).

\section{Epidermal progenitor-like cells are present} in culture-expanded rat TM cells

Culture expanded epidermal-like cells were enriched for putative stem cell markers integrin b1 and CK 19. Magnetic isolation/enrichment protocols were repeated twice and the approximate cell yield for each marker respectively was $0.9 \%$ and $2.3 \%$ cells per $1 \cdot 107$ total cells (data not shown). Cells enriched for these two putative stem cell markers were able to attach to collagen IV coated dishes and formed colonies. Integrin b1-positive cells did not survive culture expansion via trypsinization and failed to grow after cryopreservation. Meanwhile, CK 19+ cells were more robust and survived both trypsinization and cryopreservation. Similar to the unenriched rat TM epidermal cells, CK 19+ cells had a high nuclear to cytoplasm ratio and do not form tight epithelial colonies like HaCat cell lines, as shown using immunofluorescent staining in Fig. 5C.

\section{Discussion}

The spontaneous clearing and repair mechanisms inherent to the TM are thought to indicate the existence of a central "regenerative" region and a strong epidermal migration to the periphery. Cells that express putative epidermal stem cell markers have been located at the umbo region and annulus $[3,5]$. However, little is known about the characteristics and biology of TM progenitor cells either in vivo or in vitro. Using a rat model, our current study aimed to explore and refine the methodologies used to isolate TM cells ex vivo and to characterize the cells according to colony forming capacity and putative stem cell markers.

Cell surface proteins have been proposed as in vivo markers to identify human and rodent epidermal stem cells, including integrin a6, CD71, P63, and CK 15 [18-21]. In this study, we have demonstrated that integrin b1 and CK 19 were expressed uniformly in the epidermal layer of TMs. This finding is in accordance with the study by Kim and colleagues [10] but contradictory to an earlier study [3], in which cells expressing putative stem cell markers were only found at the basal layer of keratinized epithelium in the umbo and annulus of human TM. Possible explanations for this discrepancy is the difference in epidermal thickness (rat $=5-10 \mathrm{~mm}$, human 20-40 $\mathrm{mm}$ ) and cross-species variation. While it is commonly agreed that the principal regenerative centres of TM are located at the umbo and annulus region, the biology of the resident stem cells remains unclear.

The rat TM has a diameter of 2.2-2.4 mm, a surface area of $* 11 \mathrm{~mm}, 2$ and a pars tensa thickness of only $5-10 \mathrm{~mm}$ [22]. It has been a technical challenge to obtain intact rat TM due to the small size and location deep within the temporal bone, so there is a lack of established extraction protocols. In a pilot experiment, we evaluated a direct transcanal approach using alligator scissors and found this 
technique to be inferior as the surgical equipment was difficult to maneuver through the external ear canal of rats and blocked the view from the otomicroscope. Only damaged and incomplete TMs were harvested using this approach. We have then developed and refined a method to harvest intact TMs fromrat involving en bloc excision of the tympanic bulla and overnight treatment with $0.25 \%$ Dispase II, a mild, neutral protease that has been widely applied to dissociate skin epidermis fromthe underlying dermis tissue [23]. We found the layers of TM to remain intact after Dispase II treatment but there was a beneficial effect in that intact TM could be easily " "peeled" intact fromthe bulla bone and external auditory canal tissue using a pair of forceps and used to generate explant cultures.Cell origin is of utmost importance in the study of stem cells. Our current protocol allows isolation of cells specifically from the TM as other tissues were excised before explant culture. Moreover, in comparison to themethods used by Kimand colleagues [10] where they utilized trypsin and collagenase digestion of the whole TM to obtain potential stem cells, our protocol is simpler and more able to utilize specific regional areas of the TM.

In this study, we explored variations of explant cultures to better understand the ex vivo biology of TM cells. We first FIG. 5. Phenotype of rat TM epithelial cells. (A) Immunofluorescent staining of HaCat showing cobble stonelike morphology and high proliferative capabilities. (B) Rat TM epithelial cells appeared more elongated and expressed high levels of pancytokeratin and Ki-67. (C) CK 19enriched cells displayed irregular cell morphology with higher nuclear to cytoplasm ratio. Scale bars $=500 \mathrm{~mm}$. employed the standard tissue explant technique in which the peeled whole TM or excised umbo region was adhered to collagen IV coated tissue culture dish to allow outgrowth of cells. This methodology has been widely used for a variety of tissues including skin but it is not ideal in our current study for a few reasons. First, the TM is composed of a thin, conical, hydrophobic membrane attached to the handle of malleus. The conical shape allows significantly reduced contact area on the tissue culture dish, leading to low adherence and in our experience, the tissues often floated-off when culture medium was added. While some TM tissues successfully adhered to the dish and showed outgrowth of cells, the reproducibility of this method was low. We then attempted to graft excised TM umbo on the membrane of a cell culture insert [17], to provide a 3D-like surface for cells to attach and propagate. The grafted umbo served as the "'regenerative region', and cells grew in a continuous manner outward toward the periphery of the cell culture insert. This mechanism is similar to the in vivo epithelial migration pattern in both human and animals $[11,13,24]$. As compared to a standard explant, this model has better reproducibility and we were able to harvest cells from the membrane for at least three to four cycles. Moreover, this model has the potential for ex vivo testing of novel graft materials for tympanoplasty. In vivo studies from our group have previously investigated re-myringotomy as a method to delay TMP healing in rats.We found that instead re-myringotomy accelerated TMP closure and was associated with epidermal proliferation [25]. It is known that physical trauma activates cell proliferative mechanisms in vivo, and we proposed that this can be used to increase 
yield in our ex vivo explant culture. Thus, we chose to harvest theTMs at 3 days postmyringotomy as itwas within the "proliferative stage" of TMP closure as previously reported $[26,27]$. Outgrowth of a mixed cell population of fibroblasts and epidermal-like cells was seen in explant cultures from the myringotomy group as early as $24 \mathrm{~h}$. In comparison, a more homogenous outgrowth of cells with epidermal characteristic was observed in explant cultures of normal control TM.

Nevertheless, the outgrowth of cells was slower (48-72 h) in explant cultures of normal control TM, indicating a role for physical trauma in the activation of proliferative centers in the TM.This activation of regenerative processes was supported by positive staining of proliferative marker Ki-67 near the TMP edge and umbo region (Fig. 2I, J). Nevertheless, attempts to dissect the umbo region of the perforated TM for grafting were unsuccessful as TM tissues became brittle and prone to breakage.

Maintaining an on-going/robust cell culture was the goal of this study to allow further purification and characterization of the cell populations obtained from explant cultures.

Primary cells obtained using all three explant methods consisted of a mixed fibroblast and epidermal population based on cell morphology. Fibroblasts were easily omitted from the culture by reducing serum content in the medium or using selective trypsinization. Our initial aim was to create a feeder-free culture system for TM epidermal cells but we found that these cells did not proliferate well without feeders and formed loose colonies upon fibroblast removal (Fig. 3E). We attempted to reintroduce mitomycin-c treated TM fibroblasts as feeder cells but the results were unsatisfactory (data not shown). Therefore, we used a modified feeder system using mitomycin-c MEF cultured in complete KSFM supplemented with FBS. Using this system, TM epidermal cells were able to be propagated and enabled us to generate stable stock cultures for further characterization studies. We have also characterized our TM cells with functional assays including CFU and migration assays. TM epidermal cells possess clonogenicity as shown by the large colonies formed (Fig. 4A, B) and were able to migrate to close the gap created in a timely manner (Fig. 4C).

Cell sorting and enrichment protocols such as fluorescence activated cell sorting (FACS) are generally regarded as the gold standard in the isolation of stem cell populations from tissues [28], however, the method usually requires high starting cell numbers. A major difficulty with the enrichment of TM epidermal stem cells is the small cell population obtained from tissues and explant cultures. Hence, we employed a different approach by expanding our primary culture with MEF feeders before cell enrichment using a

MACS system. This system was able to produce a satisfactory cell yield from single explants so was easier and more economical to set up as compared to FACS. We were able to obtain integrin b1 and CK 19-positive epidermal cell populations. Both integrin b1 and CK 19-positive populations were able to adhere to collagen IV coated cell culture dishes but the integrin b1 cells did not survive trypsinization and cryopreservation. On the other hand, CK 19 cells were more robust and we were able to confirm their phenotype using immunofluorescent staining. 
In summary, we have investigated different methods for the isolation of rat TM primary cells. Our findings suggest that TM umbo grafted on culture well inserts to be superior as it allows consecutive, in situ cell harvest. In the present model, we found that physical acute trauma plays an important role in the activation of the proliferative centres in the TM, particularly at the umbo region. More importantly, we were able to confirm the presence of potential epidermal stem cells in rat TM and enrich the primary cells based on putative stem cell markers including CK 19 and integrin b1. The findings of this study provide a platform for tissue engineering of the TM and offers opportunities for further studies that may reveal the mechanism engaged in TM perforation healing.

\section{Acknowledgments}

This work was in part funded by Garnett Passe and Rodney Williams Memorial Foundation and National Health and Medical Research Council of Australia. The authors wish to thank Dr. Jeffrey Tzu-Yu Wang for his role in animal ethics application, Dr. Yi Shen for his guidance in animal surgery, Associate Professor Pritinder Kaur for advice on cell culture, Ms. Sandra Eu for assistance with staining of histologic sections, Dr. Lauren Callahan and Ms. Simone Ross for veterinary advice and animal house staff (Carmel McLeod, Neill Wilson, Sandra Goodin, and Michael Pethick). The authors wish to acknowledge Karl Storz Ltd. for the supply of microsurgical instruments.

Author Disclosure Statement

No competing financial interests exist.

\section{References}

1. Lim DJ. (1968). Tympanic membrane. Electron microscopic observation. I: pars tensa. Acta Otolaryngol 66:181-198.

2. Lim DJ. (1968). Tympanic membrane. II. Pars flaccida. Acta Otolaryngol 66:515-532.

3. Knutsson J, M von Unge, and H Rask-Andersen. (2011).

Localization of progenitor/stem cells in the human tympanic membrane. Audiol Neurootol 16:263-269.

4. Wang WQ, ZM Wang and FL Chi. (2004). Spontaneous healing of various tympanic membrane perforations in the rat. Acta Otolaryngol 124:1141-1144.

5. Wang WQ, ZM Wang and J Tian. (2004). [Epidermal stem cells in the tympanic membrane]. Zhonghua Er Bi Yan Hou Ke Za Zhi 39:712-716.

6. Mondain M and A Ryan. (1993). Histological study of the healing of traumatic tympanic membrane perforation after basic fibroblast growth factor application. Laryngoscope 103:312-318.

7. Mondain M and A Ryan. (1995). Epidermal growth factor and basic fibroblast growth factor are induced in guinea-pig tympanic membrane following traumatic perforation. Acta Otolaryngol 115:50-54.

8. Von Unge M and M Hultcrantz. (2011). The early events in the healing of laser-produced tympanic membrane perforation. Acta Otolaryngol 131:480-487.

9. Rahman A, P Olivius, J Dirckx, M Von Unge and M Hultcrantz. (2008). Stem cells and enhanced healing of chronic tympanic membrane perforation. Acta Otolaryngol 128:352-359.

10. Kim SW, J Kim, S Hoon, KJ Jang, YJ Kim, HJ Lim, KT Lim, C Tian, JH Chung and YH Choung. (2015). Latent progenitor cells as potential regulators for tympanic membrane 
regeneration. Sci Rep 5:11542.

11. Alberti PW. (1964). Epithelial migration on the tympanic membrane. J Laryngol Otol 78:808-830.

12. Makino K and M Amatsu. (1986). Epithelial migration on the tympanic membrane and external canal. Arch Otorhinolaryngol 243:39-42.

13. Tabacca NE, LK Cole, A Hillier and PJ Rajala-Schultz (2011) Epithelial migration on the canine tympanic membrane. Vet Dermatol 22:502-510.

14. Dominici M, K Le Blanc, I Mueller, I slaper-Cortenbach, FC Marini,DSKrause, RJ Deans,AKeating,DJ Prockop and EM Horwitz. (2006). Minimal criteria for defining multipotent mesenchymal stromal cells. The International Society for Cellular Therapy position statement. Cytotherapy 8:315-317. 15. Donati, G and FM Watt. (2015). Stem cell heterogeneity and plasticity in epithelia. Cell Stem Cell 16:465-476. 16. Redmond SL, B Levin, KA Heel, MD Atlas and RJ Marano. (2011). Phenotypic and genotypic profile of human tympanic membrane derived cultured cells. J Mol Histol 42:15-25. 17. Liew LJ, RM Day and RJ Dilley. (2017). Tympanic membrane organ culture using cell culture well inserts engrafted with tympanic membrane tissue explants. BioTechniques 62:109-114.

18. Li A and P Kaur. (2005). FACS enrichment of human keratinocyte stem cells. Methods Mol Biol 289:87-96.

19. Lyle S, M Christofidou-Solomidou, Y Liu, DE Elder, S Albelda and G Cotsarelis. (1998). The C8/144B monoclonal antibody recognizes cytokeratin 15 and defines the location of human hair follicle stem cells. J Cell Sci 111:

3179-3188.

20. Pellegrini G, E Dellambra, O Golisano, E Martinelli, I Fantozzi, S Bondanza, D Ponzin, F McKeon and M De Luca. (2001). p63 identifies keratinocyte stem cells. Proc Natl Acad Sci U S A 98:3156-3161.

21. Webb A, A Li and P Kaur. (2004). Location and phenotype of human adult keratinocyte stem cells of the skin. Differentiation 72:387-395.

22. Wang AY, Y Shen, JT Wang, PL Friedland, MD Atlas and RJ Dilley. (2014). Animal models of chronic tympanic membrane perforation: a 'time-out' to review evidence and standardize design. Int J Pediatr Otorhinolaryngol 78:20482055.

23. Kitano Y and N Okada. (1983). Separation of the epidermal sheet by dispase. Br J Dermatol 108:555-560.

24. Santhi K, N Prepageran, IP Tang and R Raman. (2014). Study of epithelial migration in the tympanic membrane and bony external auditory canal wall in patients with irradiated nasopharyngeal carcinoma. Otol Neurotol 36:318-322.

25. Wang AY, Y Shen, LJ Liew, JT Wang, M von Unge, MD Atlas and RJ Dilley. (2015). Searching for a rat model of chronic tympanic membrane perforation: healing delayed by mitomycin $\mathrm{C} /$ dexamethasone but not paper implantation or iterative myringotomy. Int J Pediatr Otorhinolaryngol 79:1240-1247.

26. Santa Maria PL, SL Redmond, RL McInnes, MD Atlas and R Ghassemifar (2011) Tympanic membrane wound healing in rats assessed by transcriptome profiling. Laryngoscope 121:2199-2213.

27. Santa Maria PL, SL Redmond, MD Atlas and R Ghassemifar. (2010). Histology of the healing tympanic membrane following perforation in rats. Laryngoscope 120:2061-2070. 28. Nowak JA and E Fuchs. (2009). Isolation and culture of epithelial stem cells. Methods Mol Biol 482:215-232.

Address correspondence to: 
Lawrence J. Liew

Ear Sciences Centre

School of Medicine (M507)

University of Western Australia

Nedlands 6009

Australia

E-mail: lawrence.liew@earscience.org.au 